The Astrophysical Journal, 655:364-374, 2007 January 20

(C) 2007. The American Astronomical Society. All rights reserved. Printed in U.S.A.

\title{
THE SPITZER c2d SURVEY OF NEARBY DENSE CORES. IV. REVEALING THE EMBEDDED CLUSTER IN B59
}

\author{
Timothy Y. Brooke, ${ }^{1}$ Tracy L. Huard, ${ }^{2}$ Tyler L. Bourke, ${ }^{2}$ A. C. Adwin Boogert, ${ }^{1,3}$ Lori E. Allen, ${ }^{2}$ \\ Geoffrey A. Blake, ${ }^{4}$ Neal J. Evans II, ${ }^{5}$ Paul M. Harvey, ${ }^{5}$ David W. Koerner, ${ }^{6}$ Lee G. Mundy, ${ }^{7}$ \\ Philip C. Myers, ${ }^{2}$ Deborah L. Padgett, ${ }^{8}$ Anneila I. Sargent, ${ }^{1}$ Karl R. Stapelfeldt, ${ }^{9}$ \\ Ewine F. van Dishoeck, ${ }^{10}$ Nicholas Chapman, ${ }^{7}$ Lucas Cieza, ${ }^{5}$ Michael M. Dunham, \\ Shith-Ping Lai, ${ }^{7,11,12}$ Alicia Porras, ${ }^{2}$ William Spiesman, ${ }^{5}$ Peter J. Teuben, ${ }^{7}$ \\ Chadwick H. Young, ${ }^{13}$ Zahed Wahhaj, ${ }^{6}$ and Chang Won LeE ${ }^{14}$ \\ Received 2006 June 13; accepted 2006 October 9
}

\begin{abstract}
Infrared images of the dark cloud core B59 were obtained with the Spitzer Space Telescope as part of the "Cores to Disks" Legacy Science project. Photometry from 3.6-70 $\mu$ m indicates at least 20 candidate low-mass young stars near the core, more than doubling the previously known population. Out of this group, 13 are located within $\sim 0.1 \mathrm{pc}$ in projection of the molecular gas peak, where a new embedded source is detected. Spectral energy distributions span the range from small excesses above photospheric levels to rising in the mid-infrared. One other embedded object, probably associated with the millimeter source B59-MMS1, with a bolometric luminosity $L_{\mathrm{bol}} \sim 2 L_{\odot}$, has extended structure at 3.6 and $4.5 \mu \mathrm{m}$, possibly tracing the edges of an outflow cavity. The measured extinction through the central part of the core is $A_{V} \gtrsim 45 \mathrm{mag}$. The B59 core is producing young stars with a high efficiency.
\end{abstract}

Subject headings: infrared: ISM — infrared: stars — ISM: clouds — stars: pre-main-sequence

\section{INTRODUCTION}

One component of the Spitzer Space Telescope "Cores to Disks" (c2d) Legacy Science program (Evans et al. 2003), is imaging of nearby dark cloud cores at infrared wavelengths ranging from 3.6 to $70 \mu \mathrm{m}$. The program takes advantage of Spitzer's high sensitivity (Werner et al. 2004) to survey young stars down to $\sim 0.001 L_{\odot}$ in these cores. Five large molecular clouds are also being studied by $\mathrm{c} 2 \mathrm{~d}$. This will allow comparisons to be made between the young star populations in isolated cores and large molecular clouds. Spitzer's high sensitivity in the mid-infrared makes it ideally suited to studying the intermediate stages of star formation, particularly in dense cores, where dust extinction can obscure the light at visible and near-infrared wavelengths.

This paper presents highlights of the c2d observational data of the dark cloud core B59. Although it is a known site of star formation, there have been relatively few published studies of this core to date and spectral information is apparently lacking for nearly all of the objects discussed here. The Spitzer data show the

\footnotetext{
1 Astronomy Department, California Institute of Technology, Pasadena, CA; tyb@astro.caltech.edu.

${ }_{2}^{2}$ Smithsonian Astrophysical Observatory, Cambridge, MA.

3 NOAO Gemini Science Center, La Serena, Chile.

4 Department of Geological and Planetary Sciences, California Institute of Technology, Pasadena, CA

${ }_{6}^{5}$ Department of Astronomy, University of Texas, Austin, TX.

${ }^{6}$ Department of Physics and Astronomy, Northern Arizona University, Flagstaff, AZ.

7 Department of Astronomy, University of Maryland, College Park, MD.

${ }^{8}$ Spitzer Science Center, California Institute of Technology, CA. CA.

9 Jet Propulsion Laboratory, California Institute of Technology, Pasadena,

${ }^{10}$ Leiden Observatory, Leiden, Netherlands.

${ }^{11}$ Institute of Astronomy and Department of Physics, National Tsing Hua University, Hsinchu, Taiwan.

12 Academia Sinica Institute of Astronomy and Astrophysics, P.O. Box 23-141, Taipei, Taiwan

${ }_{13}$ Department of Physical Sciences, Nicholls State University, Thibodaux, LA.

${ }^{14}$ Korea Astronomy and Space Science Institute, Daejeon, South Korea.
}

core to be the site of a small cluster of young low-mass stars at a range of evolutionary stages.

\section{B59}

B59 (Barnard 1927; Lynds 1962) is an irregularly shaped dark cloud roughly $30^{\prime} \times 15^{\prime}$ centered around $\alpha=17^{\mathrm{h}} 11.4^{\mathrm{m}}$ and $\delta=-27^{\circ} 26^{\prime}$ (J2000.0; Herbig 2005). It sits at the northwest end (in Galactic coordinates) of the Pipe Nebula, a set of filamentary dark clouds located close to our line of sight to the Galactic center and above the Galactic plane $\left(l=357^{\circ}, b=7.2^{\circ}\right.$ for B59). The nebula also lies close in projection to the Ophiuchus molecular cloud.

The Pipe Nebula was mapped in the $J=1-0$ lines of ${ }^{12} \mathrm{CO}$, ${ }^{13} \mathrm{CO}$, and $\mathrm{C}^{18} \mathrm{O}$ with the NANTEN telescope using a $2.7^{\prime}$ beam (Onishi et al. 1999). They identified $14 \mathrm{C}^{18} \mathrm{O}$ cores. Molecular outflow emission was detected only toward a single core (their Core 1), within B59. This core has roughly twice the column density of the other cores. The outflow was not clearly identifiable as bipolar, but may be due to two or more different outflows. We will refer to this as the B59 core, and sometimes loosely B59, in this paper.

The distance to B59 is uncertain. The radial velocity of the molecular gas is consistent with a distance similar to the Ophiuchus cloud (Onishi et al. 1999), which is approximately $d=125 \pm 25 \mathrm{pc}$ (de Geus et al. 1989). The distance to the Pipe Nebula was recently estimated by combining extinctions with parallaxes (Lombardi et al. 2006). They obtained $d=130_{-20}^{+13} \mathrm{pc}$, where the uncertainties are $1 \sigma$. This distance is adopted in this paper.

B59 is the only confirmed star-forming region in the Pipe Nebula to date. In addition to the $\mathrm{CO}$ outflow(s), there are at least five $\mathrm{H} \alpha$ emission-line stars in or near the core: $\mathrm{LkH} \alpha 346$ (NW and SE), KW 2, B59-2, and $\mathrm{LkH} \alpha 347$ (Cohen \& Kuhi 1979; Herbig \& Bell 1988; Kohoutek \& Wehmeyer 2003; Herbig 2005). Two other $\mathrm{H} \alpha$ emitters, KK Oph and V359 Oph, are farther from the core center, $\sim 20^{\prime}$ and $\sim 36^{\prime}$, or 0.8 and $1.4 \mathrm{pc}$ in projection, but may also be associated with B59 (Herbig 2005).

There are three bright IRAS sources in the Point Source Catalog within $\sim 5.5^{\prime}$ of the center of the core with spectral energy 
TABLE 1

Candidate Young Stars in B59

\begin{tabular}{|c|c|c|c|c|c|c|c|c|c|c|}
\hline \multirow[b]{2}{*}{ No. } & \multirow[b]{2}{*}{ 2MASS Name } & \multirow[b]{2}{*}{ OTHER ID } & \multirow{2}{*}{$\begin{array}{c}\text { R.A. } \\
(J 2000.0)\end{array}$} & \multirow{2}{*}{$\begin{array}{c}\text { DECL. } \\
(\mathrm{J} 2000.0)\end{array}$} & \multirow[b]{2}{*}{$J$} & \multirow[b]{2}{*}{$H$} & \multirow[b]{2}{*}{$K_{s}$} & \multicolumn{2}{|c|}{$A_{V}$} & \multirow[b]{2}{*}{ Comments } \\
\hline & & & & & & & & $J H K^{\mathrm{a}}$ & Map $^{\mathrm{b}}$ & \\
\hline $1 \ldots \ldots \ldots$ & $\mathrm{J} 17110392-2722551$ & $\mathrm{LkH} \alpha 346 \mathrm{NW}$ & 171103.91 & -272255.2 & $\begin{array}{l}10.46 \\
(0.03)\end{array}$ & $\begin{array}{c}8.99 \\
(0.03)\end{array}$ & $\begin{array}{c}7.76 \\
(0.02)\end{array}$ & $\begin{array}{c}2.6 \\
(1.7)\end{array}$ & $\begin{array}{l}10.0 \\
(0.6)\end{array}$ & $\begin{array}{l}\text { Triple }^{\mathrm{c}} \\
\text { IRAS } 17079-2719\end{array}$ \\
\hline $2 \ldots \ldots \ldots$ & $\mathrm{J} 17110411-2722593$ & $\mathrm{LkH} \alpha 346 \mathrm{SE}$ & 171104.12 & -272259.3 & $\begin{array}{c}9.75 \\
(0.03)\end{array}$ & $\begin{array}{c}8.79 \\
(0.04)\end{array}$ & $\begin{array}{c}8.05 \\
(0.03)\end{array}$ & $\begin{array}{c}0.0 \\
(1.5)\end{array}$ & $\begin{array}{c}9.9 \\
(0.6)\end{array}$ & \\
\hline $3 \ldots \ldots \ldots$ & $\mathrm{J} 17111182-2726547$ & $\ldots$ & 171111.82 & -272655.0 & $\begin{array}{l}14.09 \\
(0.02)\end{array}$ & $\begin{array}{l}12.62 \\
(0.03)\end{array}$ & $\begin{array}{l}11.76 \\
(0.02)\end{array}$ & $\begin{array}{c}5.6 \\
(1.4)\end{array}$ & $\begin{array}{l}19.2 \\
(1.9)\end{array}$ & \\
\hline $4 \ldots \ldots \ldots$ & $\mathrm{J} 17111445-2726543$ & $\cdots$ & 171114.45 & -272654.4 & $\begin{array}{l}11.62 \\
(0.02)\end{array}$ & $\begin{array}{l}10.42 \\
(0.02)\end{array}$ & $\begin{array}{c}9.63 \\
(0.02)\end{array}$ & $\begin{array}{c}2.7 \\
(1.3)\end{array}$ & $\begin{array}{l}22.4 \\
(2.8)\end{array}$ & \\
\hline $5 \ldots \ldots \ldots$ & $\mathrm{J} 17111626-2720287$ & $\cdots$ & 171116.27 & -272028.8 & $\begin{array}{l}11.23 \\
(0.02)\end{array}$ & $\begin{array}{c}9.70 \\
(0.02)\end{array}$ & $\begin{array}{c}9.07 \\
(0.02)\end{array}$ & $\begin{array}{c}8.3 \\
(0.8)\end{array}$ & $\begin{array}{c}7.3 \\
(0.3)\end{array}$ & \\
\hline $6 \ldots \ldots \ldots$ & $\mathrm{J} 17111631-2725144$ & KW 2 & 171116.32 & -272514.6 & $\begin{array}{l}10.58 \\
(0.02)\end{array}$ & $\begin{array}{c}9.37 \\
(0.02)\end{array}$ & $\begin{array}{c}8.75 \\
(0.02)\end{array}$ & $\begin{array}{c}4.2 \\
(1.1)\end{array}$ & $\begin{array}{l}14.7 \\
(2.3)\end{array}$ & $\mathrm{H} \alpha^{\mathrm{d}}$ \\
\hline $7 \ldots \ldots \ldots$ & $\mathrm{J} 17111726-2725081$ & IRAS $17081-2721$ & 171117.28 & -272508.2 & $\begin{array}{l}13.61 \\
(0.04)\end{array}$ & $\begin{array}{l}10.82 \\
(0.04)\end{array}$ & $\begin{array}{c}8.77 \\
(0.03)\end{array}$ & $\begin{array}{l}13.0 \\
(1.8)\end{array}$ & $\begin{array}{l}15.7 \\
(2.5)\end{array}$ & \\
\hline $8 \ldots \ldots \ldots$ & $\mathrm{J} 17111827-2725491$ & $\cdots$ & 171118.13 & -272549.3 & $>18.4$ & $\begin{array}{l}15.12 \\
(0.08)\end{array}$ & $\begin{array}{l}11.95 \\
(0.03)\end{array}$ & $\begin{array}{l}44 \\
(8)\end{array}$ & $\begin{array}{l}25.3 \\
(6.2)\end{array}$ & \\
\hline $9 \ldots \ldots \ldots$ & $\mathrm{J} 17112153-2727417$ & IRAS $17082-2724$ & 171121.50 & -272742.3 & $\begin{array}{l}12.74 \\
(0.02)\end{array}$ & $\begin{array}{l}10.57 \\
(0.02)\end{array}$ & $\begin{array}{c}8.98 \\
(0.02)\end{array}$ & $\begin{array}{c}8.7 \\
(1.7)\end{array}$ & $\begin{array}{l}26.9 \\
(5.0)\end{array}$ & \\
\hline $10 \ldots \ldots$ & $\cdots$ & $\cdots$ & 171122.10 & -27262.0 & $\ldots$ & $\ldots$ & $\ldots$ & $\ldots$ & $\begin{array}{c}46.1 \\
(20.0)\end{array}$ & \\
\hline $11 \ldots \ldots \ldots$ & $\mathrm{J} 17112317-2724315$ & $\cdots$ & 171123.18 & -272431.5 & $>18.8$ & $>17.8$ & $\begin{array}{c}15.08 \\
(0.14)\end{array}$ & $\cdots$ & $\begin{array}{l}25.7 \\
(5.7)\end{array}$ & Prob. MMS $1^{\mathrm{e}}$ \\
\hline $12 \ldots \ldots \ldots$ & $\mathrm{J} 17112508-2724425$ & $\cdots$ & 171125.08 & -272442.7 & $\begin{array}{l}16.52 \\
(0.11)\end{array}$ & $\begin{array}{l}13.61 \\
(0.03)\end{array}$ & $\begin{array}{l}11.57 \\
(0.02)\end{array}$ & $\begin{array}{l}14.7 \\
(2.2)\end{array}$ & $\begin{array}{l}27.2 \\
(5.7)\end{array}$ & \\
\hline $13 \ldots \ldots \ldots$ & $\mathrm{J} 17112701-2723485$ & B59-1 & 171126.95 & -272348.4 & $\begin{array}{l}11.88 \\
(0.04)\end{array}$ & $\begin{array}{l}10.14 \\
(0.04)\end{array}$ & $\begin{array}{c}9.08 \\
(0.03)\end{array}$ & $\begin{array}{c}7.5 \\
(1.5)\end{array}$ & $\begin{array}{l}24.4 \\
(2.4)\end{array}$ & Triple $^{f}$ \\
\hline $14 \ldots \ldots \ldots$ & $\mathrm{J} 17112729-2725283$ & $\cdots$ & 171127.06 & -272529.5 & $\begin{array}{l}13.17 \\
(0.02)\end{array}$ & $\begin{array}{l}10.65 \\
(0.02)\end{array}$ & $\begin{array}{c}9.11 \\
(0.02)\end{array}$ & $\begin{array}{l}13.7 \\
(1.3)\end{array}$ & $\begin{array}{l}33.2 \\
(8.6)\end{array}$ & \\
\hline $15 \ldots \ldots \ldots$ & $\mathrm{J} 17112942-2725367$ & $\cdots$ & 171129.31 & -272536.3 & $\begin{array}{l}13.28 \\
(0.02)\end{array}$ & $\begin{array}{l}11.74 \\
(0.02)\end{array}$ & $\begin{array}{l}10.70 \\
(0.02)\end{array}$ & $\begin{array}{c}5.1 \\
(1.3)\end{array}$ & $\begin{array}{l}23.6 \\
(3.7)\end{array}$ & \\
\hline $16 \ldots \ldots$. & $\mathrm{J} 17113036-2726292$ & $\ldots$ & 171130.29 & -272629.3 & $\begin{array}{l}11.91 \\
(0.03)\end{array}$ & $\begin{array}{l}10.00 \\
(0.02)\end{array}$ & $\begin{array}{c}8.89 \\
(0.02)\end{array}$ & $\begin{array}{c}9.3 \\
(1.4)\end{array}$ & $\begin{array}{l}20.7 \\
(2.7)\end{array}$ & \\
\hline $17 \ldots \ldots$. & $\mathrm{J} 17114099-2718368$ & IRAS $17085-2715$ & 171140.99 & -271837.0 & $\begin{array}{l}10.64 \\
(0.02)\end{array}$ & $\begin{array}{c}8.75 \\
(0.04)\end{array}$ & $\begin{array}{c}7.54 \\
(0.02)\end{array}$ & $\begin{array}{c}8.2 \\
(1.4)\end{array}$ & $\begin{array}{c}8.4 \\
(0.8)\end{array}$ & \\
\hline $18 \ldots \ldots$. & $\mathrm{J} 17114182-2725477$ & B59-2 & 171141.73 & -272550.3 & $\begin{array}{l}11.3 \\
(0.2)\end{array}$ & $\begin{array}{l}10.4 \\
(0.2)\end{array}$ & $\begin{array}{l}10.1 \\
(0.2)\end{array}$ & $\begin{array}{c}4.1 \\
(4.3)\end{array}$ & $\begin{array}{l}11.7 \\
(0.6)\end{array}$ & Double $^{g}$ \\
\hline $19 \ldots \ldots \ldots$ & $\mathrm{J} 17114315-2730584$ & $\ldots$ & 171143.16 & -273058.6 & $\begin{array}{l}14.09 \\
(0.03)\end{array}$ & $\begin{array}{l}12.75 \\
(0.03)\end{array}$ & $\begin{array}{l}11.91 \\
(0.03)\end{array}$ & $\begin{array}{c}4.1 \\
(1.4)\end{array}$ & $\begin{array}{l}10.1 \\
(1.2)\end{array}$ & \\
\hline $20 \ldots \ldots$ & $\mathrm{J} 17120020-2720180$ & $\mathrm{LkH} \alpha 347$ & 17120.20 & -272018.1 & $\begin{array}{l}10.54 \\
(0.02)\end{array}$ & $\begin{array}{c}9.64 \\
(0.03)\end{array}$ & $\begin{array}{c}9.17 \\
(0.02)\end{array}$ & $\begin{array}{c}1.4 \\
(1.1)\end{array}$ & $\begin{array}{c}6.9 \\
(0.8)\end{array}$ & \\
\hline
\end{tabular}

Notes.-Within field covered by both IRAC and MIPS $24 \mu \mathrm{m}$. Uncertainties in parentheses. Adopted flux zero points for the $2 \mathrm{MASS} J, H$, and $K_{s}$ filters are 1594,1024 , and $667 \mathrm{Jy}$, respectively. Units of right ascension are hours, minutes, and seconds, and units of declination are degrees, arcminutes, and arcseconds.

a Extinction from dereddening to $\mathrm{T}$ Tauri $J H K_{s}$ locus.

b Cloud extinction from background stars.

${ }^{c} \mathrm{LkH} \alpha 346 \mathrm{SE}$ is labeled the primary (A) and has a tertiary companion (C) according to Chelli et al. (1995). The AC pair is unresolved by Spitzer. LkH $\alpha$ 346 $\mathrm{NW}$, or (B), is the brighter infrared source.

d' $\mathrm{H} \alpha$ emission (Kohoutek \& Wehmeyer 2003).

e Probably MMS1 of Reipurth et al. (1996). See text.

${ }^{\mathrm{f}}$ The source is triple according to Koresko (2002). The brighter source is the primary (plus tertiary), for which the position and fluxes are given.

$\mathrm{g}$ The source is double (Reipurth \& Zinnecker 1993), possibly triple (Koresko 2002). The brighter mid-infrared source is the secondary, for which the position and fluxes are given. The $J H K$ fluxes are from Koresko (2002).

distributions consistent with young stellar objects, according to the criteria of Lee \& Myers (1999): IRAS 17079-2719 (associated with $\mathrm{LkH} \alpha$ 346), IRAS 17081-2721, and IRAS 17082-2724. Because of the proximity of the emission-line stars and IRAS sources, B59 is categorized by the c2d team as a "starred" core. A $1.3 \mathrm{~mm}$ continuum survey revealed an additional probable protostellar object, B59-MMS1 (Reipurth et al. 1996), not associated with any of the IRAS sources.

There have been several studies of B59 that have focused on the multiple stars. B59-1 and the emission-line star, $\mathrm{LkH} \alpha 346$, are probable triple systems (Chelli et al. 1995; Koresko 2002). Another emission-line star, B59-2, is at least double (Reipurth \& Zinnecker 1993; Chelli et al. 1995), and may be triple (Koresko 2002).

\section{OBSERVATIONS}

B59 was observed with the Spitzer Infrared Array Camera, IRAC (Fazio et al. 2004), on UT 2004 September 3.9 and 4.2 under Astronomical Observation Requests (AORs) 5131776 and 5132288. The pixel size is $1.2^{\prime \prime}$. Observations at two different 
TABLE 2

Spitzer Fluxes (mJy) of Candidate Young Stars in B59

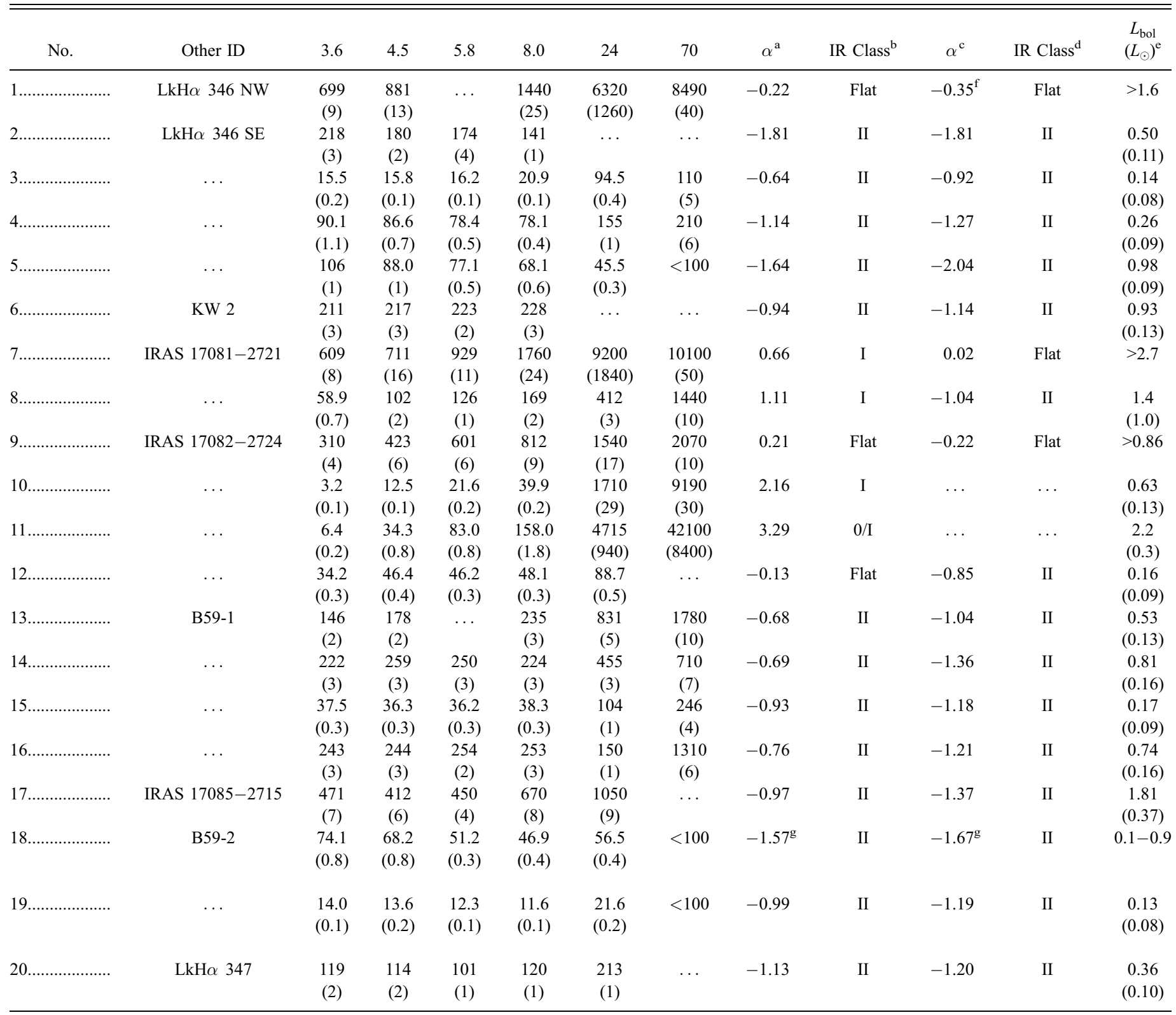

Notes.-Within field covered by both IRAC and MIPS $24 \mu \mathrm{m} .1 \sigma$ errors (in parentheses) are from photometric fitting and do not include absolute flux error (see text). A blank entry indicates no useful value due to confusion with nearby source(s), or due to lack of data.

${ }^{a}$ Spectral index $d \log \left(\lambda F_{\lambda}\right) / d \log (\lambda)$ with endpoints at 2.2 and $8.0 \mu \mathrm{m}$ uncorrected for extinction, unless different endpoint wavelengths in $\mu \mathrm{m}$ are noted.

b IR spectral class.

${ }^{c}$ Same as footnote a after correction for extinction.

${ }^{\mathrm{d}}$ Same as footnote $\mathrm{b}$ after correction for extinction.

e Bolometric luminosity (or lower limit) estimated as discussed in text. Uncertainties (in parentheses) do not include uncertainty in distance.

${ }^{\mathrm{f}}$ On border of Flat and Class II, but longer wavelengths indicate Flat.

${ }^{\mathrm{g}}$ Spectral index is from 3.6 to $8.0 \mu \mathrm{m}$.

epochs allowed identification of asteroids. The IRAC 3.6 and $5.8 \mu \mathrm{m}$ arrays observe the same field simultaneously, and the 4.5 and $8.0 \mu \mathrm{m}$ arrays a different field. The observations at each epoch consisted of a $3 \times 4$ map with small overlap regions. For each map there were two dithers of $12 \mathrm{~s}$ exposures, preceded by short highdynamic-range $0.6 \mathrm{~s}$ exposures (HDRs). The HDR observations allowed photometry of sources that saturated at longer integration times. The typical effective observation time was $48 \mathrm{~s}$. The pointsource flux limits for reliable detection were roughly $0.03,0.03$, $0.10,0.15 \mathrm{mJy}$ at $3.6,4.5,5.8,8.0 \mu \mathrm{m}$, respectively.
Observations of B59 at 24 and $70 \mu \mathrm{m}$ using the Multiband Infrared Photometer for Spitzer (MIPS; Rieke et al. 2004), were obtained on UT 2005 April 6.4 and 7.0 (AORs 9409280 and 9438720). The pixel size is $2.5^{\prime \prime}$ at $24 \mu \mathrm{m}$ and $10^{\prime \prime}$ at $70 \mu \mathrm{m}$. The observations were $3 \times 3$ photometry maps with one cycle of $3 \mathrm{~s} \mathrm{ob}$ servations at $24 \mu \mathrm{m}$, and three cycles of $3 \mathrm{~s}$ at $70 \mu \mathrm{m}$. The second epoch was shifted by roughly $6^{\prime}$, to increase coverage at $70 \mu \mathrm{m}$. Typical observation times were $96 \mathrm{~s}$ at $24 \mu \mathrm{m}$ and $100 \mathrm{~s}$ at $70 \mu \mathrm{m}$. The point-source flux limits for reliable detection were roughly $0.5 \mathrm{mJy}$ at $24 \mu \mathrm{m}$ and $100 \mathrm{mJy}$ at $70 \mu \mathrm{m}$. 
The data presented here are from the Spitzer Science Center's (SSC) pipeline version S11.4.0. The data were further processed by the $\mathrm{c} 2 \mathrm{~d}$ team to correct artifacts, where possible. This was followed by creation of new mosaics, source photometry up to $24 \mu \mathrm{m}$, bandmerging for the detected wavelengths, and preliminary source typing with extinction estimates. Counterparts in the Two Micron All Sky Survey (2MASS) catalog were assigned iffound. The procedures are described in Evans et al. (2005). Photometry at $70 \mu \mathrm{m}$ was done separately with the SSC's Mopex package, release version 030106 (Makovoz \& Marleau 2005).

One exception to these procedures is that three sources in the B59 field saturated at $24 \mu \mathrm{m}(1,7$, and 11 in Tables 1 and 2) and one source saturated at $70 \mu \mathrm{m}$ (source 11). For these sources, photometry was done by fitting the radial profile in the wings with a point-spread function obtained from an unsaturated bright point source in the field. The flux uncertainty due to this fitting is estimated to be $20 \%$.

The Spitzer photometry is summarized in Table 2. The uncertainties given do not include absolute calibration uncertainties. These are currently estimated by the SSC to be $10 \%$ for IRAC, $10 \%$ for MIPS $24 \mu \mathrm{m}$, and $20 \%$ for MIPS $70 \mu \mathrm{m}$.

The results in this paper are limited to the region where all IRAC bands and the MIPS $24 \mu \mathrm{m}$ areas overlap, about $170 \mathrm{arcmin}^{2}$. The MIPS 70 coverage is a subset of this, about $60 \%$ of the area.

\section{RESULTS}

An overview of the young star cluster in B59 is provided by the IRAC three-color image and the MIPS $24 \mu \mathrm{m}$ image in Figures $1 a$ and $1 b$. Source 10 (Fig. $1 c$ ) is near the peaks in the molecular gas and in the dust extinction, which are discussed below.

Selection of candidate young stars in B59 was based on infrared excesses relative to photospheric colors, after correction for extinction. The excess method is needed because of the absence of any systematic study of the core membership. Correction for extinction is vital due to the high dust column densities toward the core.

As part of its band-merging, c2d checks whether a reddened stellar photosphere can fit the 2MASS and Spitzer fluxes at all wavelengths available for each source, within the uncertainties. When this was successful, the source was dropped from consideration as a candidate young star here. Note that some of these may in fact be young stars, although with no detected infrared excesses.

For the remaining sources, which could be young stars, mainsequence or post-main-sequence stars with excesses, or galaxies, we imposed a $24 \mu \mathrm{m}$ flux lower limit, $F_{\nu}(24 \mu \mathrm{m})>3 \mathrm{mJy}$. This tends to cut out galaxies as the number of galaxies brighter than this is $\sim 50 \mathrm{deg}^{-2}$ (Marleau et al. 2004; Papovich et al. 2004), or $\sim 2$ in our field. ( Two of our candidates have no measured $24 \mu \mathrm{m}$ fluxes due to confusion with nearby sources, but their expected $24 \mu \mathrm{m}$ fluxes would easily make this cut.)

The Galactic background counts toward B59 are less certain. The model of Wainscoat et al. (1992) indicates that for $F_{\nu}(24 \mu \mathrm{m})>$ $3 \mathrm{mJy}$, the number of nonphotospheric Galactic sources, primarily O-rich and C-rich asymptotic giant branch (AGB) stars, should be small, $\sim 13 \mathrm{deg}^{-2}$, or $\sim 0.6$ in our field. But models can serve only as a guide for small areas.

Lombardi et al. (2006) identified a population of AGB stars behind the Pipe Nebula from their location in the 2MASS colorcolor diagram. These are bright objects, peaking at $K \sim 7$, most likely in the Galactic bulge. If, as suggested by Lombardi et al., they are similar to the OH/IR star sample of Jiménez-Esteban et al. (2005), then they would be easily detectable at $24 \mu \mathrm{m}$,
$F_{\nu}(24 \mu \mathrm{m}) \gtrsim 1 \mathrm{Jy}$. But they are too few in number to significantly contaminate the young star candidate sample; at $b=7.2^{\circ}$, there are $\sim 3 \mathrm{deg}^{-2}$, or $\sim 0.14$ in our field.

To be conservative, we also cut out sources with excesses that appeared close to photospheric levels. We required at least one flux to be a factor of $\sim 4$ higher than the expected photosphere at that wavelength. This eliminated four more objects. In doing so, we may have dropped some young stars with weak dust excesses. The motivation is that $\mathrm{c} 2 \mathrm{~d}$ is finding that there are some sources with small IR excesses toward the Serpens cloud that are background AGB stars.

The 20 candidate young stars are listed in Tables 1 and 2. Positions are shown in Figure $1 c$. Note that 13 of the objects lie within $\sim 0.1 \mathrm{pc}$ in projection of the molecular gas peak (see Fig. 1). We cannot completely eliminate the possibility that some of the proposed young stars are background objects without further data. So we refer to the set as candidate young stars. But some have $\mathrm{H} \alpha$ in emission $(\S 2)$, and the majority are clustered together close to the center of the core.

For the candidate young stars, we re-evaluated their extinction taking into account the possibility of excess emission in the near-IR. There is no systematic visible photometry of the cloud members, nor is there information on spectral types or effective temperatures for most of the objects. We rely on typical dereddened near-IR colors of young stars.

We assumed that the young stars with all three 2MASS fluxes in our sample have intrinsic $J H K_{s}$ colors that fall on the locus of the sample of T Tauri stars in Taurus, whose dereddened colors were estimated by Meyer et al. (1997); see Figure 2. This requires that we are sampling similar spectral types and dust properties as in Taurus. It is difficult to test this without spectroscopic data on the stars. But the assumption has been used for other young clusters, e.g., IC 348 (Muench et al. 2003) and NGC 2024 (Haisch et al. 2001), in the absence of better constraints. And Luhman (2004) shows consistency of extinction estimates done this way compared to those obtained from visible spectra for young stars in Cham I.

We adopted the dust extinction law of Indebetouw et al. (2005), which extends from $1.25-8.0 \mu \mathrm{m}$. The $1.25-2.2 \mu \mathrm{m}$ extinction of the Pipe Nebula agrees with that of Indebetouw et al. within the uncertainties (Lombardi et al. 2006). Using this dust extinction law, we allowed the young stars to deredden to the locus of $J H K_{s}$ colors of the T Tauri stars, or to the nearest extrapolated point (Fig. 2). Uncertainties from both the data and the locus envelope are propagated to the extinction estimate (Table 1). By convention, the extinction is given by $A_{V}$ in magnitudes, whereas we do not know the extinction law in this region down to visible wavelengths. We only estimate near-infrared values of the extinction, e.g., $A_{K}$. We converted using $A_{V} / A_{K}=8.9$ from the Rieke \& Lebofsky (1985) extinction law, but note that our actual estimates are $A_{K}$. The adopted Indebetouw et al. extinction law appears appropriate for B59 in the $J H K_{s}$ bands, as the bright stars in the field follow it (Fig. 2).

For source 8 , with only $H K_{s}$ data, we deredden to the center of the $\mathrm{T}$ Tauri locus and assign a larger uncertainty. The embedded objects $(10,11)$ have insufficient near-IR data to correct for extinction by this method.

The dereddened spectral energy distributions (SEDs) are shown in Figure 3. The $24 \mu \mathrm{m}$ extinction, $A_{24} / A_{K}=0.24$, was taken from the Weingartner \& Draine (2001) model which fits the Indebetouw et al. points $\left(R_{V}=5.5\right.$, Case $\left.\mathrm{B}\right)$.

The $2-10 \mu \mathrm{m}$ or $2-20 \mu \mathrm{m}$ slope is commonly used to classify young stars in the IR. Following earlier authors (Lada 1987; Wilking et al. 2001; Greene et al. 1994) we define the spectral 

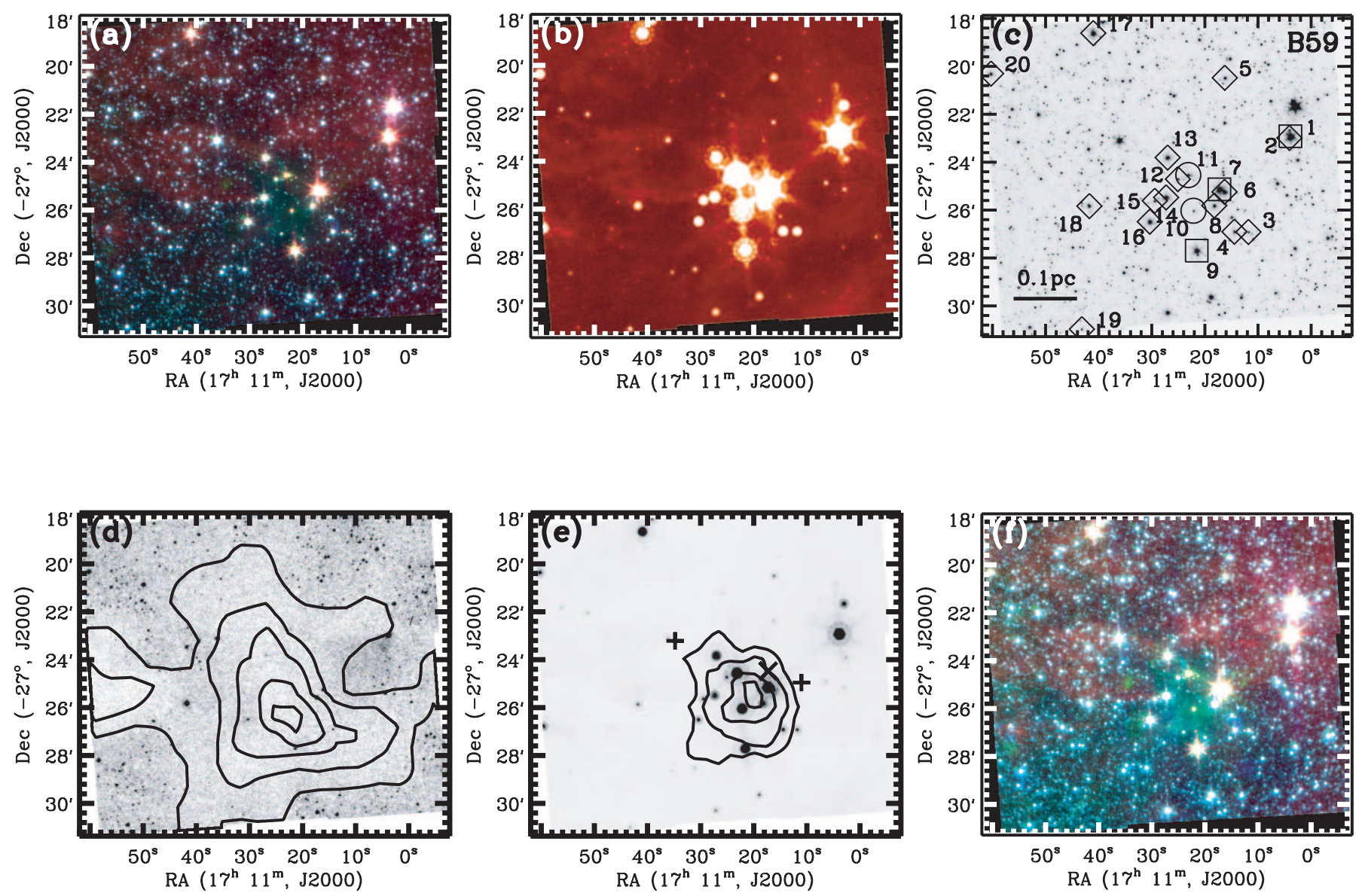

FIG. 1.-Multiwavelength views of the B59 region. (a) Three-color composite in IRAC filters: $3.6 \mu \mathrm{m}$ (blue), $4.5 \mu \mathrm{m}$ ( green), and $8.0 \mu \mathrm{m}($ red $)$. (b) MIPS $24 \mu \mathrm{m}$ image revealing the young star cluster. Non-starlike features seen $\pm 2^{\prime}$ roughly north and south of bright objects are artifacts due to latent images. (c) Source ID key for candidate young stars from Table 1, with IRAC $4.5 \mu \mathrm{m}$ image in background. Young star IR spectral classes have the following symbols: Class I (circles), Flat (squares), and Class II (diamonds). The scale bar is for adopted distance of $d=130$ pc. $(d)$ Estimated $A_{V}$ from 2MASS data with Digital Sky Survey Red image. Extinction map has resolution $\sim 100^{\prime \prime}$ and contours at $A_{V}=10,14,19,30$, and 45 mag. (e) MIPS $24 \mu$ m image with CS $(J=2-1)$ integrated intensity contours with a 60" beam from C. DeVries (2006, private communication). Contours are at $0.36,0.50,0.70$, and $0.85 \mathrm{~K} \mathrm{~km} \mathrm{~s}^{-1}$. CO red outflow (dark cross), and CO blue outflow peaks (dark plus signs) are from Onishi et al. (1999). These are the peak positions of the integrated line flux in red and blue channels. A third blue CO outflow peak is off the map to the SE. $(f)$ Same as $(a)$, but stretched to show extended emission.

index as $\alpha=d \log \left(\lambda F_{\lambda}\right) / d \log \lambda$. We define the IR spectral classes as follows: $\alpha \geq 0.3$ is a Class I source, $0.3>\alpha \geq-0.3$ a "Flat" type, and $\alpha<-0.3$ a Class II. These agree with Greene et al. (1994), except that the Class III designation is restricted to sources with practically photospheric slopes after dereddening, $\alpha \sim-3$ (Kenyon \& Hartmann 1995). Because of our selection criteria, we have no Class III sources in the present sample. We use the 2MASS $K_{s}$ and IRAC $8 \mu \mathrm{m}$ fluxes as endpoints, unless noted in Table 2. Slopes and IR class are given in Table 2 for the observed fluxes uncorrected for extinction and with correction for extinction. (Source 1 after correction is on the border of Flat and Class II, but the complete SED indicates it is most like the Flat objects.)

The similarity of the dereddened SEDs to known young stars in other star-forming regions gives us confidence that the $A_{V}$ values are reasonable and that all of the objects are young stars. Note in particular (Fig. 3) the strong similarity of most of the Class II sources with $\alpha \geq-1.6$ to the median Taurus Class II SED from the sample defined by D'Alessio et al. (1999).

\subsection{Extinction Map}

There are sufficient numbers of background stars detected through the B59 region to provide an approximate extinction map. The $H-K$ color excess was adopted as a measure of extinction
(Lada et al. 1994). This is an effective technique as the spread in $H-K$ colors of main-sequence stars is small $(\sim 0.3 \mathrm{mag})$. We require 2MASS colors at present, so some highly extincted background stars (for which we could in principle estimate $A_{V}$ from Spitzer colors alone) could not be used. But there are enough 2MASS sources for a map, and the accuracy and wide use of this method led us to adopt it.

We assumed a mean intrinsic color of $H-K=0.175$ for the background stars, which is the mean value for stars in the Pipe Nebula control field of Lombardi et al. (2006), obtained from M. Lombardi (2006, private communication). The conversion to $A_{V}$ as described above is $A_{V}=16.8 E(H-K)$, where $E(H-K)$ is the color excess. The factor 16.8 differs from the commonly used 15.9, due to the adoption of the Indebetouw et al. (2005) near-IR extinction law. Note that the maximum uncertainty in intrinsic color translates into an uncertainty in $A_{V}$ for an individual star of $2.5 \mathrm{mag}$, which is small enough to not affect our results.

The spatial resolution of the extinction map was set by the need for several stars in each spatial bin. This required boxes of size $\sim 100^{\prime \prime}$, and the map was oversampled by a factor of 2 . The median $A_{V}$ in each bin was taken to avoid foreground, objects and the scatter gave the uncertainty. The extinction map is shown in Figure $1 d$, and interpolated values at the positions of the candidate young stars in Table 1 . 


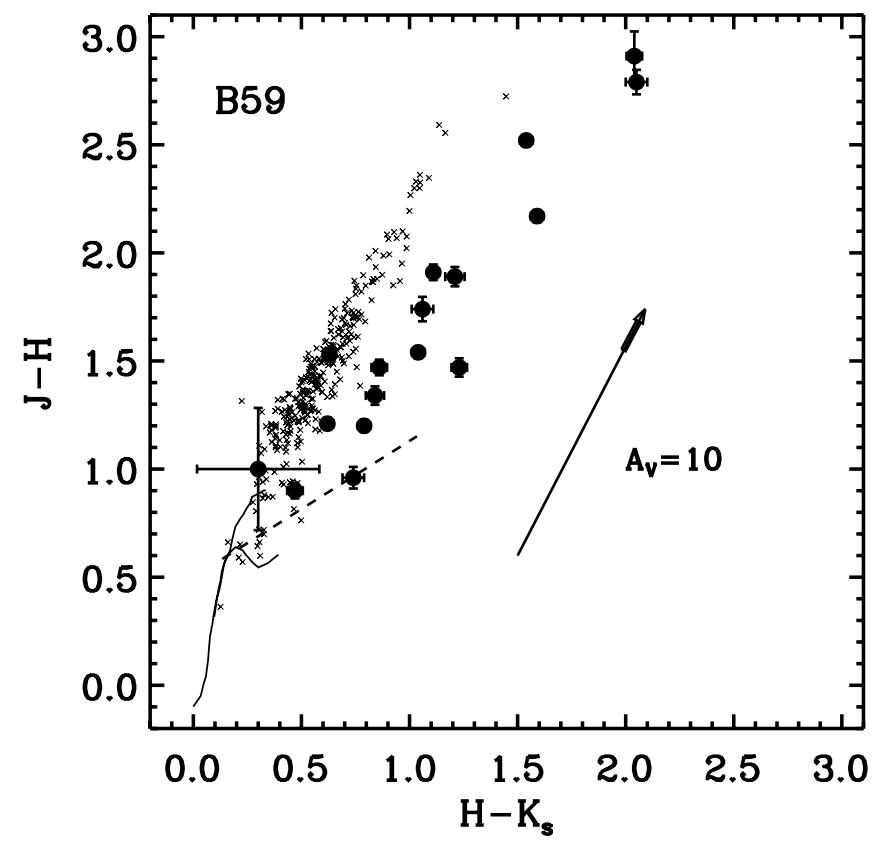

FIG. 2.-2MASS $J H K_{s}$ fluxes of candidate young stars in B59 ( filled circles) and objects with $J$ flux $\gtrsim 2.5 \mathrm{mJy}$ and classified as stars by c2d (small crosses). Main sequence and giants from Bessell \& Brett (1988) are represented by the solid lines, and the Taurus T Tauri main locus from Meyer et al. (1997) is represented by the dashed line. Both are converted to the 2MASS system using Carpenter (2001). The reddening arrow shows the adopted extinction law (Indebetouw et al., 2005) converted to $A_{V}$ (see text) and with $A_{V}=10$ mags.

The agreement between the total extinction $A_{V}$ estimates and those toward individual young stars is not particularly good. Most of the extinction map $A_{V}$ values are higher than those derived by dereddening individual objects. This is reasonable since many of the objects could be near the front of the cloud. Two are lower, which may be explained by localized dust.

The measured peak extinction from the background stars is $A_{V} \sim 45 \mathrm{mag}$, although the true value is likely higher due to the coarse spatial resolution. We estimated the molecular hydrogen column density at the peak using $N\left(\mathrm{H}_{2}\right)=9.4 \times 10^{20} A_{V}$ (Kandori et al. 2005), giving $N\left(\mathrm{H}_{2}\right)=4.2 \times 10^{22} \mathrm{~cm}^{-2}$ with a $50 \%$ uncertainty. For the $\mathrm{C}^{18} \mathrm{O}$ core in B59, Onishi et al. (1999) estimated $N\left(\mathrm{H}_{2}\right)=1.5 \times 10^{22} \mathrm{~cm}^{-2}$ from the strength of the $\mathrm{C}^{18} \mathrm{O}$ line.

\subsection{Comparison to Gas}

In addition to the $\mathrm{CO}$ data of Onishi et al. (1999), data for B59 in CS $(J=2-1)$ and $\mathrm{N}_{2} \mathrm{H}^{+}(J=1-0)$ have been obtained at FCRAO with an effective beam size of $60^{\prime \prime}$ (C. DeVries 2007, in preparation). CS integrated intensity contours are shown in Figure $1 e$. Source 10 lies close to the CS molecular gas peak. The $\mathrm{N}_{2} \mathrm{H}^{+}$emission (not shown) is centered near this area, but appears extended northeast-southwest, i.e., similar to the extinction. No strong, well-organized outflow or infall signatures were detected for either species in these data.

The positions of the $\mathrm{CO}$ outflows within our mapped region from Onishi et al. (1999) are marked in Figure 1e. These are the positions of the peak contours in the red and blue channels defined by those authors. However the large beam size of these observations $\left(2.7^{\prime}\right)$ makes detailed comparison to the Spitzer sources difficult. It appears that some of the $\mathrm{CO}$ outflows are aligned with source 11 , rather than source 10 , which is closer to the extinction peak (see $\S 4.4)$.
The total gas mass in the B59 core estimated from the $\mathrm{C}^{18} \mathrm{O}$ line is $\sim 20 M_{\odot}$ within a radius of $\sim 0.15$ pc (Onishi et al. 1999, both adjusted for the different assumed distances).

The total gas mass in the core can also be estimated from the dust extinction of background stars under the assumption that dust within the core provides most of the extinction. The conversion from $A_{V}$ to $N\left(\mathrm{H}_{2}\right)$ for typical interstellar dust is given above. For a standard cloud composition with mean atomic weight per $\mathrm{H}$ atom, $\mu=1.37$ (Lombardi et al. 2006), the total core mass integrated within the confines of the $\mathrm{C}^{18} \mathrm{O}$ core is $M_{\text {core }}=25 \pm 1 M_{\odot}$, where the uncertainty does not include the distance uncertainty.

These two estimates of core gas mass are in good agreement, considering the different spatial resolutions. Some freeze-out of $\mathrm{CO}$ is probable in the dense regions. Although solid $\mathrm{CO}$ has not yet been observed toward B59, sources near the center of the core have deep solid $\mathrm{H}_{2} \mathrm{O}$ and $\mathrm{CO}_{2}$ bands (A. Boogert 2007, in preparation).

\subsection{Class 0/I and I Sources and Bolometric Luminosities}

Based on their SED's there are two deeply embedded objects near the center of the B59 core. Source $11=2$ MASS J171123172724315 (hereafter 2MASS 171123) lies $\sim 15^{\prime \prime}$ east of the quoted position of the $1.3 \mathrm{~mm}$ source B59-MMS1 observed with a $22^{\prime \prime}$ half-power beamwidth beam (Reipurth et al., 1996) and the two are probably the same. The total $1.3 \mathrm{~mm}$ flux integrated to the $8 \sigma$ contour was $725 \mathrm{mJy}$. It was recently detected at $350 \mu \mathrm{m}$ with the SHARCII bolometer array at the Caltech Submilllimeter Observatory ( Wu et al. 2006). The flux in a $40^{\prime \prime}$ diameter aperture is $45.2 \pm 8.0 \mathrm{Jy}$.

With most of the SED for this source in hand (Fig. 4), we estimated the bolometric luminosity, $L_{\text {bol }}=2.2 \pm 0.3 L_{\odot}$, the bolometric temperature, $T_{\text {bol }}=70 \pm 10 \mathrm{~K}$ (Myers \& Ladd 1993), and the ratio of submillimeter $(\gtrsim 350 \mu \mathrm{m})$ to bolometric luminosity $\left(L_{\mathrm{smm}} / L_{\mathrm{bol}}=0.03 \pm 0.01\right)$. The uncertainty in luminosity does not include the distance uncertainty.

In addition to the Class I-III spectral classes for young stars discussed above, a fourth class, Class 0 , is used to define cold sources. Two common criteria for a Class 0 source are that $T_{\text {bol }}<70 \mathrm{~K}$ and $L_{\text {smm }} / L_{\text {bol }}>0.5 \%$ (André et al. 2000).

2MASS 171123 lies somewhere near the border of this definition, so may be described as a Class $0 /$ I source.

As noted by Young et al. (2004) and others, heating of dust by the interstellar radiation field may contribute to the fluxes of lowluminosity protostars longward of $\sim 100 \mu \mathrm{m}$. Thus high values of $L_{\mathrm{smm}} / L_{\text {bol }}$ may be common for low-luminosity protostars at some point during their lifetime, and some revision of this criterion may be needed.

The other nominal Class I object, source 10, is close to the gas and dust extinction peaks. It lacks a complete SED at present, but appears spectrally similar to source 11 in the mid-IR, so we estimate a bolometric luminosity by scaling, $L_{\text {bol }} \sim 0.6 L_{\odot}$, but this is only a crude estimate. We will leave its designation as Class I for the present time, pending mm and submillimeter observations.

We are not able to determine directly the final stellar masses for these two Class 0/I or I souces. The models of Myers et al. (1998) suggest that source 11 and source 10 will evolve to form stars with 0.5 and $0.3 M_{\odot}$, and have ages of $t \sim 0.1-0.2 \mathrm{Myr}$.

It is probable that one or both of the embedded sources near the center of the core we detect with Spitzer are the youngest objects and drive the CO outflows seen by Onishi et al. (1999). Further study of these objects and their possible disks and outflows with IR and $\mathrm{mm}$ and submillimeter interferometry is needed.

Bolometric luminosities (Table 2) for the rest of the candidate young stars were estimated as follows. For the Class II sources with 


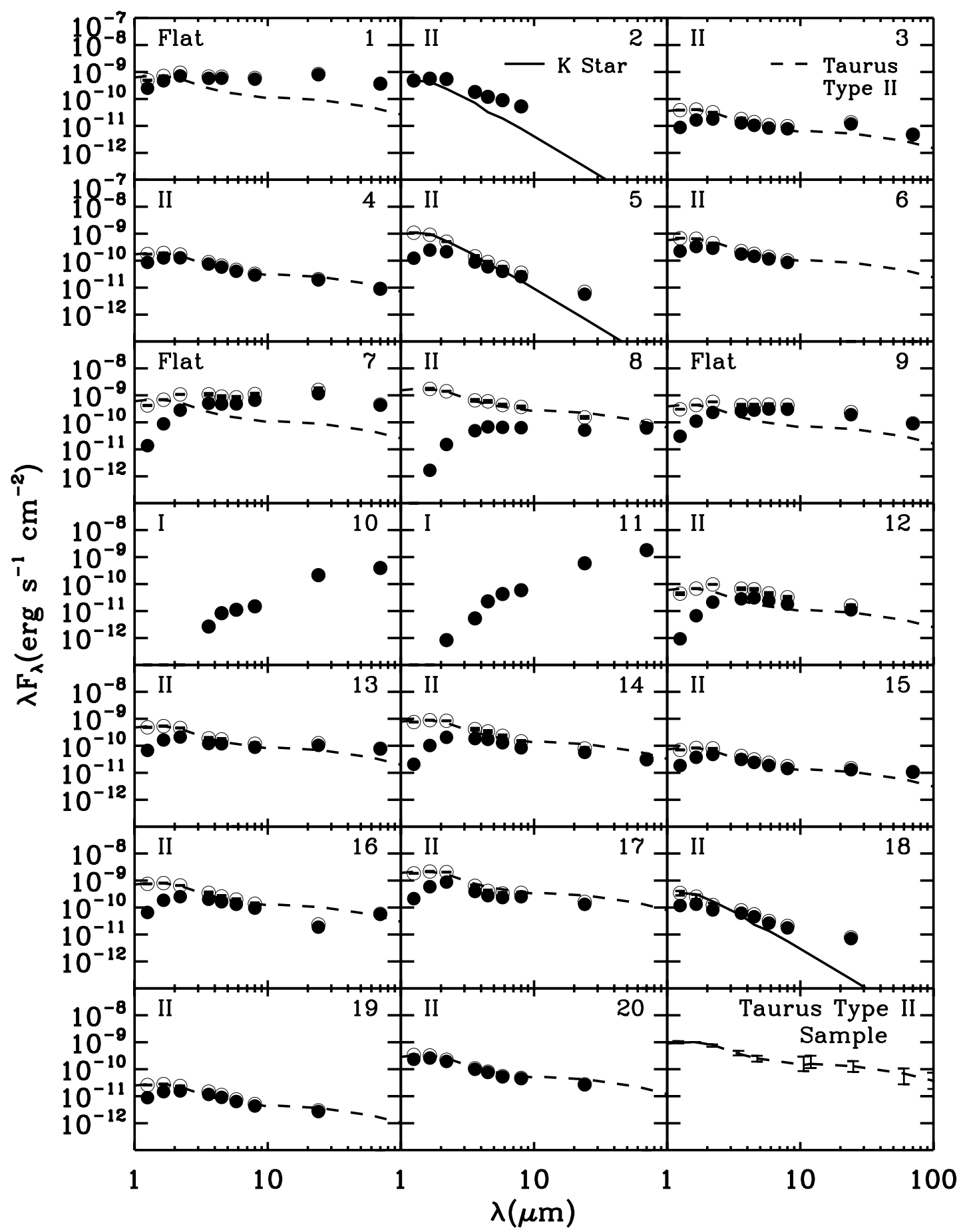

FIG. 3.-SEDs of the candidate young stars in B59 as observed ( filled circles) and after correction for extinction as described in text (open circles). Error bars, including absolute calibration uncertainties, are shown, but are smaller than the symbols. In each object panel, the source number (Table 1) is in the top right and the IR spectral class after correction for extinction, where possible, is in the top left. The two Class I sources are not corrected for extinction. For the Flat and Class II sources with $\alpha \geq-1.6$, the median Class II SED of Taurus sources (bottom right box, D'Alessio et al. 1999), scaled at H (1.65 $\mu \mathrm{m})$, is plotted for comparison (short-dashed lines). Class II sources with $\alpha<-1.6$ have a $\mathrm{K} 7$ photosphere scaled at $J(1.25 \mu \mathrm{m})$ (solid lines).

$\alpha<-1.6$, late-type photospheres were fitted to the dereddened $J$-band fluxes. The IR excesses add only marginally to the bolometric luminosity. For the Class II sources with $\alpha \geq-1.6$, we used the absolute $J$-band flux versus bolometric luminosity relation from Greene et al. (1994). For the Flat sources, we have no good estimate; only lower limits were obtained by integration under the observed fluxes.

Estimated bolometric luminosities for the Class II sources range from $0.1-1.8 L \odot$. The central stars provide the bulk of the contri- bution to the bolometric luminosity for these objects. Stellar evolutionary models (e.g., D’Antona \& Mazzitelli 1994; Siess et al. 2000) show that for plausible ages of $t=0.5-1.0 \mathrm{Myr}$, the stellar masses are $\sim 0.1-0.9 M_{\odot}$, corresponding to mid $\mathrm{G}$ to late-M dwarfs. There are no clear brown dwarf candidates in the present sample.

\subsection{Extended Structure around 2MASS 171123}

The Class 0/I source 2MASS 171123 has extended structure at 3.6 and $4.5 \mu \mathrm{m}$ (Fig. 5), extending out $\gtrsim 30^{\prime \prime}(0.02 \mathrm{pc})$ to the 


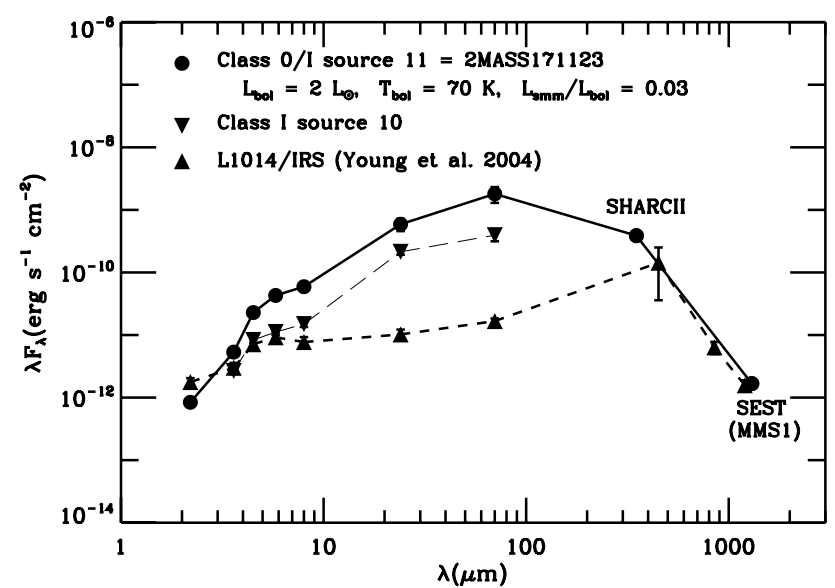

FIG. 4.- SEDs of the candidate Class 0/I or I protostars in B59, compared to the infrared source in L1014. For 2MASS 171123, the SHARCII $350 \mu \mathrm{m}$ data point is from Wu et al. (2006). The Swedish-ESO Submillimetre Telescope $1.3 \mathrm{~mm}$ data point of MMS1 is from Reipurth et al. (1996). See text for details.

northeast and southeast. There is a trace of extension at $5.8 \mu \mathrm{m}$ also. These arcs may trace the edges of an outflow cavity in reflected light. The morphology suggests a disk inclined to the line of sight. A possible third extension to the west is confused with a superposed background source.

The apparent outflow axis defined by the east lobes is $\sim 50^{\circ}$ east of north. This lines up with some of the $\mathrm{CO}$ outflow peaks (Onishi et al. 1999; see Fig. 1e). But the low spatial resolution of the $\mathrm{CO}$ data makes a firm identification of specific outflows with this source difficult.
The arcs are unlikely to be due to $\mathrm{H}_{2}$ emission, because the Spitzer IRS spectrum of the object shows no evidence of $\mathrm{H}_{2}$ emission lines at longer wavelengths (A. Boogert 2007, in preparation).

\subsection{Extended Emission}

The Spitzer images of B59 show two kinds of extended emission above the background zodiacal emission.

First is the red $(8.0 \mu \mathrm{m})$ emission seen most clearly in the northwestern area of Figure $1 f$, roughly $0.8 \mathrm{MJy} \mathrm{sr}^{-1}$ above background. This is due to Galactic cirrus dust emission. The same structure is seen in the IRAC $5.8 \mu \mathrm{m}$ band near the detection limit (not shown). The band ratio is $I(5.8) / I(8) \approx 0.25$. The emission at $24 \mu \mathrm{m}$ also broadly traces the same area, with $I(24) / I(8) \approx 0.4$. These flux ratios are in the range expected for galactic cirrus (e.g., Reach et al. 2004), with the $8 \mu$ m flux due mostly to the emission in the strong aromatic hydrocarbon bands at 7.7 and $8.6 \mu \mathrm{m}$.

The second extended structure is more interesting: a wide greenish glow centered on the core, due to emission in the IRAC $4.5 \mu \mathrm{m}$ band with a contribution from the blue $3.6 \mu \mathrm{m}$ band. This emission is just visible in Figure $1 f$ (it may be easier to see in the online version). The band ratio is roughly $I(3.6) / I(4.5)=0.75$. This emission is not detected at longer wavelengths. The coincidence of the emission with the cloud core is an indication that this is due to scattered Galactic light, sometimes called cloudshine (Foster \& Goodman 2006).

Cloudshine has been seen in many dense cloud cores at visible (Witt \& Stephens 1974) and near-IR wavelengths (Lehtinen \& Mattila 1996; Nakajima et al. 2003; Foster \& Goodman 2006). The JHK data of Foster \& Goodman (2006) have been used to study dust structure on subarcsecond scales (Padoan et al. 2006). It is reasonable to expect cloudshine at longer wavelengths as
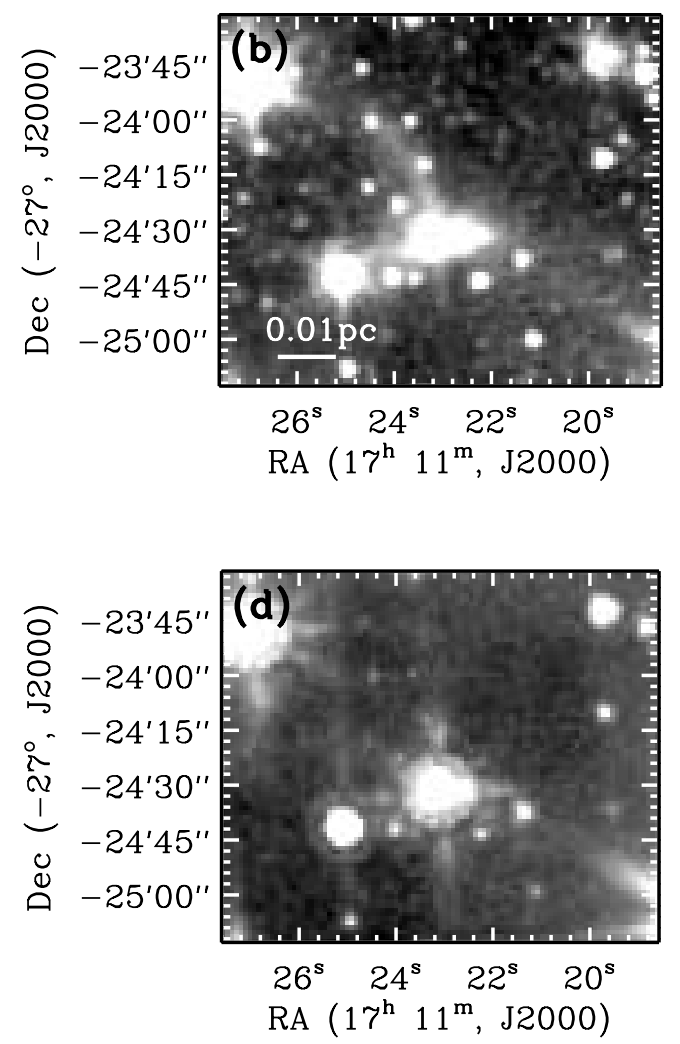

FIG. 5.-Close-up of the Class 0/I source $11=2$ MASS 171123 , closest to center, through the four IRAC bands: $(a) 3.6 \mu \mathrm{m},(b) 4.5 \mu \mathrm{m},(c) 5.8 \mu \mathrm{m}$, and $(d) 8.0 \mu \mathrm{m}$. Image arcs visible at 3.6 and $4.5 \mu \mathrm{m}$ may trace an outflow cavity in scattered light. Scale bar plotted in $(b)$ is for assumed distance of $d=130$ pc. 
well, although typical interstellar grains will have decreased scattering efficiency at longer wavelengths. Longer wavelengths will in principle penetrate deeper into the cloud, but the visibility will depend on the sensitivity of the observations and the strength of the ambient Galactic light.

This explanation is favored over $\mathrm{H}_{2}$ line emission because of the lack of an obvious jet or shock morphology, and because no $\mathrm{H}_{2}$ lines are seen in the IRS spectrum of source 10 (A. Boogert 2007 , in preparation).

\section{DISCUSSION}

The Spitzer data reveal the dark cloud core B59 to be a region of active low-mass star formation containing a small cluster of young stars at different evolutionary stages. Some authors (e.g., Lada \& Lada 2003) prefer to reserve the word cluster for groups of $\geq 35$ objects, but we use the term in a general sense here. Some notable aspects and limitations of the Spitzer observations are discussed in this section.

Completeness for weak excesses.-The sensitivity of this analysis to young stars with weak dust excesses is limited by the $24 \mu \mathrm{m}$ flux cutoff of $3 \mathrm{mJy}$. A hypothetical young star at $A_{V}=10$ with a factor of 4 excess at $24 \mu \mathrm{m}$ would not make the cut if it's unextincted photosphere had a flux below $1 \mathrm{mJy}$. For a mainsequence object at $d=130 \mathrm{pc}$, this corresponds to a mid-K dwarf. For a more likely age of $t \sim 1 \mathrm{Myr}$, this corresponds to an object that will become roughly a mid-M dwarf (Baraffe et al. 2002). Later spectral types make the cut if they have more substantial excesses. So the sample defined here may be incomplete in Class II for late spectral types with weak excesses.

IRAC color-color plot.-Many Spitzer studies of young stars are possible with IRAC alone, e.g., Hartmann et al. (2005). Figure 6 shows an IRAC color-color plot for the candidate young stars in B59 for comparison to IRAC studies of other star-forming regions. The colors plotted are as observed, although the IR spectral classes indicated are after correction for extinction, where possible. Table 2 has the IR spectral classes before correction for extinction. The adopted magnitude zero points were $278 \mathrm{Jy}(3.6 \mu \mathrm{m})$, $180 \mathrm{Jy}(4.5 \mu \mathrm{m}), 117 \mathrm{Jy}(5.8 \mu \mathrm{m})$, and $63.1 \mathrm{Jy}(8.0 \mu \mathrm{m})$.

Disk properties and age.-We have as yet no direct measure of age (e.g., Li absorption lines) for any of the candidate young stars. The IR spectral classes give a rough measure of age, with Class I being the youngest and Class III the oldest (Kenyon \& Hartmann 1995).

Many authors use a two-class definition for the IR spectral classes: $\alpha>0$ for Class I, $\alpha<0$ for Class II. For comparison in the next paragraph, B59 has a two-class Class I to Class II ratio of $3: 17$, or $18 \%$.

The two-class Class I/Class II ratio of $18 \%$ can be compared to Spitzer results on other young clusters: $17 \%-34 \%$ for the $\operatorname{Tr} 37$ globule ( $t \sim 1$ Myr; Sicilia-Aguilar et al. 2006; Reach et al. 2004); $25 \%$ for NGC 7129 ( $t \sim 1$ Myr; Muzerolle et al. 2004); and 33\% for the Spokes cluster in NGC 2264 ( $t \sim 0.5 \mathrm{Myr}$; Teixeira et al. 2006). For two clusters in Perseus, Jorgensen et al. (2006) find $29 \%$ for NGC $1333(t \lesssim 1 \mathrm{My})$ and $15 \%$ for the older IC 348 $(t \sim 2$ Myr $)$.

The above comparisons should be taken only as suggestive due to several factors: some studies do no extinction corrections; there are different sensitivities for different young star selection techniques; and sensitivities differ because of different cloud distances.

We can also use an argument based on the predicted lifetimes of embedded and T Tauri phases (Myers et al. 1987; Kenyon et al. 1990) to estimate the onset of star formation in B59. For

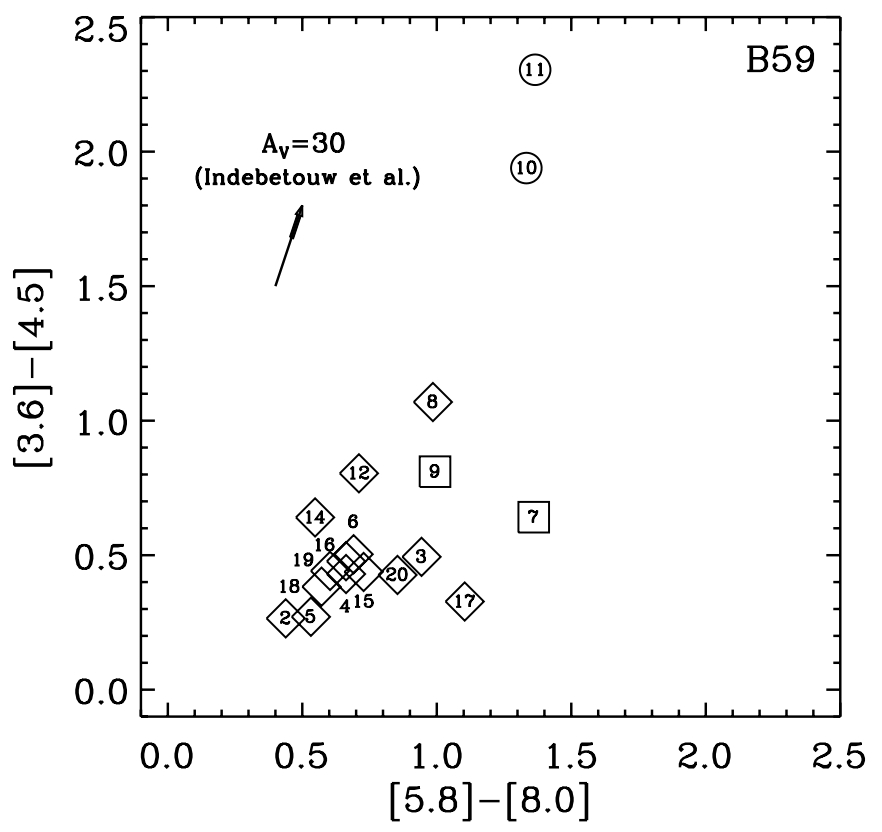

FIG. 6.-IRAC color-color diagram, as observed, of candidate young stars in B59 with source numbers from Table 1. Young star IR spectral classes, after correction for extinction (where possible) are indicated with the following symbols: Class I (circle), Flat (square), and Class II (diamond). The reddening arrow shows the adopted extinction law (Indebetouw et al., 2005) for $A_{V} \approx 30$ mags (see text).

a fixed star formation rate of objects that evolve from Class I $\left(t_{\text {life }} \sim 10^{5} \mathrm{yr}\right)$ to Class II $\left(t_{\text {life }} \sim 10^{6} \mathrm{yr}\right)$, the relative numbers suggest an age of $\sim 0.7 \times 10^{6} \mathrm{yr}$. Clearly the number of objects in each bin in B59 makes this argument only approximate. And we have neglected potentially important factors, e.g., the effect of the stellar environment on disk lifetimes.

Multiplicity also complicates the interpretation. The suspected young star pair resolved with Spitzer, LkH $\alpha 346$ NW and SE (itself a probable double), have very different SEDs (our IR spectral classes are Flat and Class II, respectively). If coeval, the simple model sketched above is wrong. In addition, the frequency of disks may be a function of spectral type, as indicated by a recent Spitzer study of IC 348 (Lada et al. 2006).

In Figure 3 we compare the SEDs of the B59 Class II objects with $\alpha \geq-1.6$ to a median of a sample of mostly Class II objects in Taurus (D'Alessio et al. 1999). The Taurus SED can be reasonably fitted by optically thick disks with flaring at large radii (D'Alessio et al. 1999). With time, disks are expected to clear from inside-out due to dust coagulation into planetesimals and dynamical clearing. Evidence for such transition disks has recently been shown for IC 348 ( $t \sim 2$ Myr; Lada et al. 2006) and $\operatorname{Tr} 37$ ( $t \sim 4$ Myr; Sicilia-Aguilar et al. 2006). The fact that most of the SEDs of the Class II sources with $\alpha \geq-1.6$ in B59 are similar to the Taurus SED suggests a comparable age for the cloud, $t \sim 0.7$ Myr (Kenyon \& Hartmann 1995).

We see no clear "transition objects," such as CoKu Tau/4 (Hartmann et al. 2005), i.e., those that have very little excess in the IRAC bands, then a large $24 \mu \mathrm{m}$ excess.

These comparisons taken together suggest an age for B59 of between 0.5 and 1 Myr. Deep optical and near-IR photometric and spectroscopic surveys of the cloud are needed to ensure the membership and measure more accurate age indicators.

Star formation in B59.- - To date, B59 is the only known starforming cloud in the Pipe Nebula. Onishi et al. (1999) suggest that this is due to its proximity to the $\mathrm{B} 0 \mathrm{star} \tau \mathrm{Oph}$, with a stellar wind having triggered star formation. 
It is notable that the star formation efficiency of B59 is relatively high. Taking just the objects near the core, there are 13 young stars in $\sim 25 M_{\odot}$ of dense gas, or $0.52 M_{\odot}{ }^{-1}$. Of the $179 \mathrm{C}^{18} \mathrm{O}$ cores in nearby star-forming regions covered in the NANTEN survey, only a few have a comparable efficiency ( Tachihara et al. 2002 and references therein). By way of contrast, the Taurus cloud as a whole has roughly 200 young stars in $\sim 10^{4} M_{\odot}$ of gas (Kenyon \& Hartmann 1995; Lada et al. 1993).

Assuming an average mass for the young stars in B59 of $0.5 M_{\odot}$, the star formation efficiency as defined by Lada \& Lada (2003), $M_{\text {star }} /\left(M_{\text {star }}+M_{\text {gas }}\right)=0.21$, close to the maximum of $\sim 0.3$ seen in their survey of young stellar clusters.

The surface density of young stars in the inner core region of B59 is $\sim 200 \mathrm{pc}^{-2}$. This is a lower limit to the young star population as we require infrared excess for inclusion. The surface density is greater than those of $90 \%$ of the young clusters tabulated in Lada \& Lada (2003), although not as high as much more massive clusters such as S106 and the Trapezium. However, the B59 core is smaller than most of the Lada \& Lada sample. Comparison to the NANTEN core sample cited above again reveals only a few with comparable young-star surface density. Recent c2d observations of another small cluster, L1251B, give a comparable stellar density to B59 (Lee et al. 2006).

Compared to all of the cores in the NANTEN CO survey (Tachihara et al. 2002), B59 is notable for its high gas column density, higher than nearly all of the starless cores and cores forming single stars, but similar to their cluster-forming cores ( $\gtrsim 10$ stars), consistent with our survey findings of a small cluster in formation.

As noted by Lada \& Lada (2003), our inventory of young, embedded star clusters, even within a few hundred parsecs, is still incomplete. Data from the Spitzer Space Telescope are helping to complete that inventory.

\section{SUMMARY}

1. At least 20 candidate young stars in the B59 core have been identified from infrared excesses in Spitzer 3.6-70 $\mu \mathrm{m}$ observations. Spectral energy distributions including corrections for extinction are presented. They range from small excesses above photospheric levels to rising in the mid-infrared. The SEDs of most of the Class II spectral types with spectral index $\alpha \geq-1.6$ are similar to the SED of the Class II sample in the Taurus cloud of D'Alessio et al. (1999).

2. Thirteen of the young star candidates are located within $\sim 0.1$ $\mathrm{pc}$ in projection of the molecular gas and dust extinction peak. Two Class 0/I or I sources are identified. One, 2MASS J17112317, probably associated with the millimeter source B59-MMS1, with a bolometric luminosity $L_{\text {bol }} \sim 2 L_{\odot}$, has extended structure at 3.6 and $4.5 \mu \mathrm{m}$, possibly tracing the edges of an outflow cavity. The other is near the molecular gas and extinction peak and previously unrecognized.

3. The measured extinction through the central part of the B59 core is $A_{V} \gtrsim 45 \mathrm{mag}$. There are extended infrared emission regions due to galactic cirrus emission and scattered Galactic light.

4. Although further study of the young star population in B59 is needed, the data to date suggest the B59 core is producing young stars with high efficiency.

We thank M. Lombardi (ESO) for assistance, C. DeVries (CSU Stanislaus) for making molecular line data available prior to publication, and R. Gutermuth (CfA) for display scripts. We thank the referee whose comments inproved the paper. This work is based on observations made with the Spitzer Space Telescope, which is operated by the Jet Propulsion Laboratory, California Institute of Technology, under NASA contract 1407. Support for this work, part of the Spitzer Space Telescope Legacy Science Program, was provided by NASA through contract numbers 1224608 , 1230779, and 1230780 issued by the Jet Propulsion Laboratory, California Institute of Technology, under NASA contract 1407. Astrochemistry in Leiden is supported by a NWO Spinoza grant and a NOVA grant. C. W. L. acknowledges suport from KOSEFF grant R01-2003-000-10513-0. This publication makes use of data products from the Two Micron All Sky Survey, which is a joint project of the University of Massachusetts and the Infrared Processing and Analysis Center/California Institute of Technology, funded by NASA and NSF. This research has made use of the SIMBAD database, operated at CDS, Strasbourg, France.

\section{REFERENCES}

André, P., Ward-Thompson, D., \& Barsony, M. 2000, in Protostars and Planets IV, ed. V. Mannings, A. P. Boss, \& S. S. Russell (Tucson: Univ. Arizona Press), 59

Baraffe, I., Chabrier, G., Allard, F., \& Hauschildt, P. 2002, A\&A, 382, 563

Barnard, E. E. 1927, Catalogue of Dark Objects in the Sky (Publ. 247, Part I; Washington: Carnegie Institute of Washington)

Bessell, M. S., \& Brett, J. M. 1988, PASP, 100, 1134

Carpenter, J. M. 2001, AJ, 121, 2851

Chelli, A., Cruz-Gonzalez, I., \& Reipurth, B. 1995, A\&AS, 114, 135

Cohen, M., \& Kuhi, L. V. 1979, ApJS, 41, 743

D’Alessio, P., Calvet, N., Hartmann, L., Lizano, S., \& Canto, J. 1999, ApJ, 527, 893

D’Antona, F., \& Mazzitelli, I. 1994, ApJS, 90, 467

de Geus, E. J., de Zeeuw, P. T., \& Lub, J. 1989, A\&A, 216, 44

Evans, N. J., et al. 2003, PASP, 115, 965

2005, Delivery of Data from the c2d Legacy Project: IRAC and MIPS

(Pasadena: SSC), http://ssc.spitzer.caltech.edu/legacy/original.html

Fazio, G. G., et al. 2004, ApJS, 154, 10

Foster, J. B., \& Goodman, A. A. 2006, ApJ, 636, L105

Greene, T. P., Wilking, B. A., André, P., Young, E. T., \& Lada, C. J. 1994, ApJ, 434, 614

Haisch, K. E., Lada, E. A., Pina, R. K., Telesco, C. M., \& Lada, C. J. 2001, AJ,

121,1512

Hartmann, L., et al. 2005, ApJ, 629, 881

Herbig, G. H. 2005, AJ, 130, 815

Herbig, G. H., \& Bell, K. R. 1988, Lick Obs. Bull. 1111

Indebetouw, R., et al. 2005, ApJ, 619, 931
Jiménez-Esteban, F. M., Agudo-Mérida, L., Engels, D., \& García-Lario, P. 2005, A\&A, 431, 779

Jorgensen, J., et al. 2006, ApJ, 645, 1246

Kandori, R., et al. 2005, AJ, 130, 2166

Kenyon, S. J., \& Hartmann, L. 1995, ApJS, 101, 117

Kenyon, S. J., Hartmann, L. W., Strom, K. M., \& Strom, S. E. 1990, AJ, 99, 869

Kohoutek, L., \& Wehmeyer, R. 2003, Astron. Nachr., 324, 437

Koresko, C. D. 2002, AJ, 124, 1082

Lada, C. J. 1987, in IAU Symp. 115, Star-Forming Regions, ed. M. Peimbert \& J. Jugaku (Dordrecht: Reidel), 1

Lada, C. J., \& Lada, E. A. 2003, ARA\&A, 41, 57

Lada, C. J., Lada, E. A., Clemens, D. P., \& Bally, J. 1994, ApJ, 429, 694

Lada, E. A., Strom, K. M., \& Myers, P. C. 1993, in Protostars and Planets III, ed. E. H. Levy \& J. I. Lunine (Tucson: Univ. Arizona Press), 245

Lada, C. J., et al. 2006, AJ, 131, 1574

Lee, C. W., \& Myers, P. C. 1999, ApJS, 123, 233

Lee, J.-E., et al. 2006, ApJ, 648, 491

Lehtinen, K., \& Mattila, K. 1996, A\&A, 309, 570

Lombardi, M., Alves, J., \& Lada, C. J. 2006, A\&A, 454, 781

Luhman, K. L. 2004, ApJ, 602, 816

Lynds, B. T. 1962, ApJS, 7, 1

Makovoz, D., \& Marleau, F. R. 2005, PASP, 117, 1113

Marleau, F. R., et al. 2004, ApJS, 154, 66

Meyer, M. R., Calvet, N., \& Hillenbrand, L. A. 1997, AJ, 114, 288

Muench, A. A., et al. 2003, AJ, 125, 2029

Muzerolle, J., et al. 2004, ApJS, 154, 379

Myers, P. C., Adams, F. C., Chen, H., \& Schaff, E. 1998, ApJ, 492, 703 
Myers, P. C., Fuller, G. A., Mathieu, R. D., Beichman, C. A., Benson, P. J., Schild, R. E., \& Emerson, J. P. 1987, ApJ, 319, 340

Myers, P. C., \& Ladd, E. F. 1993, ApJ, 413, L47

Nakajima, Y., et al. 2003, AJ, 125, 1407

Onishi, T., Kawamura, A., Abe, R., Yamaguchi, N., Saito, H., Moriguchi, Y., Mizuno, A., Ogawa, H., \& Fukui, Y. 1999, PASJ, 51, 871

Padoan, P., Juvela, M., \& Pelkonen, V.-M. 2006, ApJ, 636, L101

Papovich, C., et al. 2004, ApJS, 154, 70

Reach, W. T., et al. 2004, ApJS, 154, 385

Reipurth, B., Nyman, L.Å., \& Chini, R. 1996, A\&A, 314, 258

Reipurth, B., \& Zinnecker, H. 1993, A\&A, 278, 81

Rieke, G. H., \& Lebofsky, M. J. 1985, ApJ, 288, 618

Rieke, G. H., et al. 2004, ApJS, 154, 25
Sicilia-Aguilar, A., et al. 2006, ApJ, 638, 897

Siess, L., Dufour, E., \& Forestini, M. 2000, A\&A, 358, 593

Tachihara, K., Onishi, T., Mizuno, A., \& Fukui, Y. 2002, A\&A, 385, 909

Teixeira, P. S., et al. 2006, ApJ, 636, L45

Wainscoat, R. J., Cohen, M. J., Volk, K., Walker, H. J., \& Schwartz, D. E. 1992, ApJS, 83, 111

Weingartner, J. C., \& Draine, B. T. 2001, ApJ, 548, 296

Werner, M. W., et al. 2004, ApJS, 154, 1

Wilking, B. A., Bontemps, S., Schuler, R. E., Greene, T. P., \& André, P. 2001, ApJ, 551, 357

Witt, A. N., \& Stephens, T. C. 1974, AJ, 79, 948

Wu, J., et al. 2006, ApJ, submitted

Young, C. H., et al. 2004, ApJS, 154, 396 